EGU2020-7903

https://doi.org/10.5194/egusphere-egu2020-7903

EGU General Assembly 2020

(c) Author(s) 2021. This work is distributed under

the Creative Commons Attribution 4.0 License.

\title{
Are locked-in or living deltas more at risk?
}

Maria J. Santos, Martin O. Reader, and Stefan C. Dekker

University of Zurich, Geography, Geography, Zurich, Switzerland (maria.j.santos@geo.uzh.ch)

Delta systems are growingly at risk from ongoing global changes, sediment dynamics, and face many pressures from both upstream land use change and downstream sea level rise. Historically, deltas have been key locations for human occupation, and currently hold over 340 million inhabitants globally. This continuing pressure and risks have led deltas to become locked-in, likely losing their ability to provide ecosystem services and resilience to global change. To assess whether global deltas ranging from living to locked-in have lost their resilience to changes, we (i) used historical HYDE data to reconstruct the development of population and land use in 48 major deltas over the last 310 years, (ii) determine whether deltas are in locked in states, and (iii) assess whether locked-in deltas are more at risk due to relative sea level rise (RSLR), hazards, anthropogenic condition, investment deficit, and provision of nature contributions to people. $46 \%$ of the analyzed deltas are living deltas ( 22 out of 48 ), i.e. yet to be locked-in. Of the locked-in deltas (26 out of 48$), 21 \%(n=10)$ emerge due to engineered natural infrastructure as the development of cropland and irrigation and $33 \%(n=16)$ are locked-in due to institutional infrastructure, such as no development of population or agricultural land uses. We find that average risk index is higher for locked-in deltas due to natural infrastructure changes (higher hazard and anthropogenic risks for cropland development and higher investment deficit risk for irrigation development). Surprisingly, the most at risk deltas to future RSLR are the ones locked-in due to institutional changes and living deltas. Many locked-in deltas may be even more at risk when considering their current ability to supply regulating and material ecosystem services/nature contributions to people. Our results suggest that locked-in deltas might be more at risk from current pressures, due to a reduction in the functioning of the natural processes that govern deltas, while deltas locked-in due to social and institutional infrastructure will be more at risk in the future. Further work is necessary to understand whether these trends can be reversed. 